\title{
Rapid and Sustained Eyelid Elevation in Acquired Blepharoptosis with Oxymetazoline $0.1 \%$ : Randomized Phase 3 Trial Results
}

\author{
Jason Bacharach' \\ David L Wirta ${ }^{2}$ \\ Robert Smyth-Medina ${ }^{3}$ \\ Michael S Korenfeld (D) ${ }^{4}$ \\ Shane R Kannarr ${ }^{5}$ \\ Shane Foster $\mathbb{D D}^{6}$ \\ Mark J Jaros ${ }^{7}$ \\ Charles B Slonim ${ }^{8}$ \\ 'North Bay Eye Associates, Petaluma, \\ CA, USA; ${ }^{2}$ Aesthetic Eye Care Institute \& \\ Eye Research Foundation, Newport \\ Beach, CA, USA; ${ }^{3}$ North Valley Eye \\ Medical Group, Mission Hills, CA, USA; \\ ${ }^{4}$ Comprehensive Eye Care, Ltd, \\ Washington, MO, USA; ${ }^{5}$ Kannarr Eye \\ Care, Pittsburg, KS, USA; ${ }^{6}$ Athens Eye \\ Care, Athens, OH, USA; ${ }^{7}$ Summit \\ Analytical, Denver, CO, USA; \\ ${ }^{8}$ Department of Ophthalmology, \\ University of South Florida Morsani \\ College of Medicine, Tampa, FL, USA
}

Correspondence: Jason Bacharach North Bay Eye Associates, 104 Lynch Creek Way, Suite I5, Petaluma, CA, 94954, USA

Tel +l (707) 762-3573

Fax +I (707) 762-6873

Email jbacharach@northbayeye.com
Purpose: Oxymetazoline $0.1 \%$ is a novel ophthalmic agent for the treatment of acquired blepharoptosis in adults that has been shown to improve upper eyelid elevation and superior visual field deficits. This analysis characterized the rapid onset of upper eyelid elevation with once-daily oxymetazoline $0.1 \%$ and durability of this effect over 42 days.

Materials and Methods: Pooling data from two prospective, randomized, placebocontrolled, phase 3 studies, change in marginal reflex distance 1 (MRD-1) was evaluated at a range of post-instillation time points on treatment days 1, 14, and 42. Onset of effect was assessed beginning at 5 minutes post-administration (one study) and through 6 hours at the first two visits (both studies). Overall, 203 subjects received oxymetazoline $0.1 \%$ and 101 received vehicle.

Results: Oxymetazoline $0.1 \%$ demonstrated a rapid onset of action on all days evaluated. Mean changes from baseline 5 and 15 minutes post-oxymetazoline $0.1 \%$ instillation on day 1 were $0.59 \pm 0.72 \mathrm{~mm}$ and $0.93 \pm 0.81 \mathrm{~mm}$, respectively (vs $0.20 \pm 0.57 \mathrm{~mm}$ and $0.32 \pm$ $0.64 \mathrm{~mm}$ with vehicle; both $\mathrm{p}<0.001)$. On day 14 , mean changes from baseline 5 and 15 minutes post-oxymetazoline $0.1 \%$ instillation were $0.77 \pm 0.85 \mathrm{~mm}$ and $1.11 \pm 0.92 \mathrm{~mm}$, respectively (vs $0.42 \pm 0.78 \mathrm{~mm}$ and $0.41 \pm 0.83 \mathrm{~mm}$ with vehicle; both $\mathrm{p}<0.05$ ). This effect was also observed immediately post-instillation on day 42, where mean increases 5 and 15 minutes post-oxymetazoline $0.1 \%$ instillation were $0.86 \pm 0.85 \mathrm{~mm}$ and $1.04 \pm 0.91 \mathrm{~mm}$, respectively (vs $0.42 \pm 0.80 \mathrm{~mm}$ and $0.47 \pm 0.93 \mathrm{~mm}$ with vehicle; both $\mathrm{p}<0.005$ ). Significant improvements vs vehicle $(\mathrm{p}<0.001)$ were also observed at $2-6$ hours on days 1 and 14 . At all time points, the proportion of subjects showing a positive response to treatment $(>0 \% \mathrm{MRD}$ 1 increase) was $>15 \%$ greater in the oxymetazoline $0.1 \%$ group (range $16.6-36.1 \%$ more responders vs vehicle), with the largest differences observed 2 and 6 hours post-instillation.

Conclusion: Oxymetazoline $0.1 \%$ provided rapid and sustained upper eyelid elevation. Together with data demonstrating superior visual field improvement and a favorable safety profile, this analysis supports oxymetazoline $0.1 \%$ as an effective non-surgical treatment for acquired ptosis.

Keywords: alpha adrenergic agonist, eye drop, Müller's muscle, non-surgical, topical, pharmacologic

\section{Plain Language Summary}

Acquired blepharoptosis is a common condition that is defined by drooping of one or both upper eyelids. This drooping of the eyelids can affect appearance and vision. Until recently, the only effective treatment available for this condition was surgery. More recently, an eye drop containing the drug oxymetazoline (in $0.1 \%$ solution) has been developed for the 
treatment of acquired blepharoptosis. In this pooled analysis of data from two studies, people with acquired blepharoptosis were enrolled and split into two treatment groups. One group used oxymetazoline $0.1 \%$ eye drops, and the other used placebo eye drops, once per day for 42 days. To evaluate the effect of treatment, the position of the upper eyelids was measured at various times on treatment days 1,14 , and 42 . This analysis expands the current knowledge about oxymetazoline $0.1 \%$ eye drops by showing that treatment with this drug produced a significant change in upper eyelid elevation as soon as 5 minutes after administration on days 1, 14, and 42 (assessed in one study). Analysis on treatment days 1 and 14 showed a long-lasting effect. The analysis also showed that more people had a response to treatment (ie, experienced measurable eyelid lift) with oxymetazoline $0.1 \%$ than with placebo drops. The results of this analysis further our knowledge about oxymetazoline $0.1 \%$, the first eye drop approved for acquired blepharoptosis, and provide more evidence for its potential as a therapy.

\section{Introduction}

Blepharoptosis is a congenital or acquired condition characterized by an abnormal unilateral or bilateral drooping of the upper eyelid that can impair or even completely occlude the superior visual field. ${ }^{1}$ It is a common disorder of the eyelid, and studies of its prevalence in multiple populations reveal overall incidence rates ranging from $4.7 \%$ to $13.5 \%$ in adults, as well as sharply increasing prevalence with age. ${ }^{2-4}$ A study in the UK reported that ptosis prevalence increased from $2.4 \%$ in patients aged $50-59$ years to $20.8 \%$ in patients $\geq 70$ years old, ${ }^{2}$ while a study in Iran found an increase in prevalence from $3.1 \%$ in patients $45-49$ years old to $7.1 \%$ in patients $65-69$ years old, ${ }^{3}$ and another in a large Korean patient population reported an increase in prevalence from 5.4\% in patients $40-49$ years old to $32.8 \%$ in patients 70 years or older. ${ }^{4}$ The most common cause of acquired ptosis is stretching, dehiscence, or disinsertion of the levator muscle complex related to aging (aponeurotic ptosis). ${ }^{5-8}$

Even in mild cases, ptosis can disrupt the superior visual field, and by limiting activities of daily living, negatively impact health-related quality of life (QoL). ${ }^{9-12}$ Similarly, patient-reported outcomes reveal that ptosis is associated with appearance-related distress and dysfunction, as well as increased levels of both depression and anxiety. ${ }^{13}$ The standard of care for ptosis is surgical intervention, which is effective in improving the visual field and QoL, ${ }^{14}$ but may also be associated with complications $^{6}$ and risks of under- or over-correction that can necessitate surgical revision. ${ }^{6,15}$ Surgery also requires time for referral, consultation, the procedure itself, and recovery. Thus, a non-surgical treatment option might be beneficial for patients who do not qualify for, or are unwilling or unable to undergo ptosis correction surgery.

Oxymetazoline $\mathrm{HCl}$ ophthalmic solution $0.1 \%$ (oxymetazoline $0.1 \%$; Upneeq $^{\circledR}$, RVL Pharmaceuticals, Inc., Bridgewater, NJ, USA) is a novel ophthalmic agent recently approved for the treatment of acquired blepharoptosis in adults. The active agent, oxymetazoline, is a direct-acting $\alpha$-adrenergic agonist ${ }^{16}$ commonly used in $0.05 \%$ solution as an over-the-counter (OTC) nasal decongestant, ${ }^{17,18}$ and previously available in $0.025 \%$ concentration as an OTC eye drop to reduce hyperemia of the eye. ${ }^{19,20}$ When applied topically to the eye, oxymetazoline $0.1 \%$ is thought to raise the upper eyelid by causing contraction of Müller's muscle, which is sympathetically innervated $^{21}$ and expresses $\alpha$-adrenergic receptors. ${ }^{22}$ The possibility that administration of an $\alpha$-adrenergic agent might improve ptosis is also supported by the wellknown effect of phenylephrine in transiently decreasing ptosis. $^{23}$

Oxymetazoline $0.1 \%$ has been shown to be safe and effective for the treatment of acquired ptosis in two randomized, double-masked, placebo-controlled phase 3 clinical studies. In these trials, administration of oxymetazoline $0.1 \%$ once daily in both eyes resulted in significant improvements on the primary and secondary endpoints: superior visual field deficits and upper eyelid elevation on treatment days 1 (6 hours post-instillation) and 14 ( 2 hours post-instillation). ${ }^{24}$ The objective of this analysis was to further characterize the effect of oxymetazoline $0.1 \%$ on upper eyelid elevation in these studies, with a focus on the onset of effect after administration, as well as maintenance of the effect over 42 days of use.

\section{Materials and Methods Study Design and Population}

The design of, as well as primary and secondary efficacy and safety data from, the two double-masked, placebo controlled, multicenter phase 3 studies (Study RVL-1201-201 [NCT02436759]; Study RVL-1201-202 [NCT03565887]) included in this analysis has been previously described. ${ }^{24}$ Both studies were conducted in accordance with the principles of the Declaration of Helsinki. The studies enrolled subjects at 16 and 27 sites, respectively, in the United States. Briefly, subjects with acquired ptosis and superior visual deficit in at least one eye were randomized (2:1) to 
receive oxymetazoline $0.1 \%$ or vehicle, self-administered as one drop, once daily in the morning, in both eyes, for 42 days. Study protocols and informed consent documentation were approved by a central institutional review board (Alpha IRB, San Clemente, California) prior to study initiation. All subjects completed informed consent form prior to participation in any study-related procedures. For minors, the subject's parent or legal guardian provided written permission on behalf of the subject, and the subject provided assent, per institutional review board guidelines. All subjects, investigators, staff, and other study personnel were masked with respect to treatment identity until after final lock of the database. An independent biostatistician created the randomization scheme, using a block design. Study sites accessed the Interactive Web Response System to randomize subjects to study treatment and to assign study medication. Drug kit and randomization numbers were recorded in subject electronic case report forms. Study rationale, methodology, results, and conclusions are reported in accordance with Consolidated Standards of Reporting Trials (CONSORT) guidelines.

Subjects were eligible to enroll if they were $\geq 9$ years old (Studies RVL-1201-201 and RVL-1201-202 enrolled subjects $\geq 18$ years old and $\geq 9$ years old, respectively) and had acquired ptosis, as defined by loss of $\geq 8$ points seen in the top 2 rows on Leicester Peripheral Field Test (LPFT) and marginal reflex distance 1 (MRD-1) of $2 \mathrm{~mm}$ or less in the study eye. Pseudoptosis, congenital ptosis, Marcus Gunn jaw-winking syndrome, Horner syndrome, mechanical ptosis, myasthenia gravis, dermatochalasis extending $<3 \mathrm{~mm}$ above the upper eyelid margin (ie, redundant eyelid skin within $3 \mathrm{~mm}$ of the margin), ptosis due to any other cause, and history of ptosis repair surgery or periocular neurotoxin injection $<3$ months prior to enrollment were all grounds for exclusion. Ptosis was not characterized further post-enrollment. Given the study inclusion criteria, the majority of enrolled subjects were $\geq 18$ years old, with a single subject $<18$ years old enrolled and randomized to treatment with vehicle.

\section{Marginal Reflex Distance I (MRD-I) Analysis}

The treatment protocol, schedule of study visits, and assessments for both studies have been previously described. ${ }^{24}$ Briefly, visits were carried out for screening and on treatment days 1, 14, and 42 (or upon early termination). The current analysis extends previously reported results by evaluating the change in MRD-1 (the distance between the center of the pupillary light reflex and the upper eyelid margin with the eye in primary gaze) at additional time points over multiple days, with a focus on onset of action on treatment days 1, 14, and 42. In Study RVL-1201-201, MRD-1 was evaluated at screening, treatment day 1 (baseline, 2 and 6 hours post-instillation), treatment day 14 (pre-instillation, 2 and 6 hours postinstillation), and treatment day 42 (at study site, following at-home instillation). MRD-1 was also evaluated at these time points in Study RVL-1201-202, as well as at additional times more closely following drop instillation (5 minutes and 15 minutes post-instillation on treatment days 1, 14, and 42). As reported by Slonim et al, ${ }^{24}$ MRD-1 measurements in both studies were made from digital photographs taken at the defined time points, with significant care to ensure consistent ambient lighting for photography at all sites. Subjects were asked to relax their facial muscles, and the photograph framed the subject's face from mid-forehead to the tip of the nose vertically and from ear-to-ear horizontally. A standardized self-adhesive millimeter-scale ruler was placed vertically on the subject's forehead, centered above the eyebrows for consistency across photographs. MRD-1 and pupil diameter were measured by investigators at each study site from the digital image or color printed copy of the photograph using a handheld caliper and millimeter ruler label as the legend. Changes in MRD-1 over the course of treatment were assessed relative to pre-instillation baseline values on treatment day 1. Responders to treatment were identified post hoc, based on presence of a positive increase in MRD-1 of $>0 \%$ ("any response"), $\geq 50 \% \quad$ (" $\geq 50 \%$ response"), or $\geq 90 \%$ (" $\geq 90 \%$ response") relative to preinstillation baseline on treatment day 1 .

\section{Statistical Methods}

Determination of sample size and detailed statistical methods used in analyzing pooled change from baseline data for the two randomized studies included in this report have been previously described. ${ }^{24}$ Analyses were conducted using each study's full analysis population (all subjects receiving $\geq 1$ dose of treatment). Descriptive statistics for observed values and changes from baseline for efficacy endpoints were tabulated by visit and treatment group, and differences between treatment groups compared using analysis of covariance (ANCOVA) model with treatment and study as fixed factors and baseline score as a covariate. Treatments were compared via pairwise comparison from the model; all statistical tests 
were two-sided with p-value $<0.05$ considered statistically significant. Analyses were conducted using $\mathrm{SAS}^{\circledR}$ version 9.4 (SAS Institute, Cary, NC).

\section{Results}

\section{Study Population and Disposition}

A total of 203 patients were randomized to receive oxymetazoline $0.1 \%$ and 101 patients received vehicle in two 6-week phase 3 trials. Study RVL-1201-201 included 94 and 46 subjects who received oxymetazoline $0.1 \%$ and vehicle, respectively, and Study RVL-1201-202 included 109 and 55 subjects who received oxymetazoline $0.1 \%$ and vehicle, respectively. Subject disposition and baseline characteristics are described in detail by Slonim et al. ${ }^{24}$ Baseline clinical and demographic characteristics, including baseline mean MRD-1 (oxymetazoline 0.1\%: $1.09 \pm 0.70 \mathrm{~mm}$ vs vehicle: $1.05 \pm 0.69$ ), were similar between the pooled treatment groups, as well as between individual studies. ${ }^{24}$ Per the inclusion criteria requiring MRD-1 of $2 \mathrm{~mm}$ or less in at least one eye, baseline MRD-1 was indicative of a subject population with moderate to severe ptosis, for which surgical intervention would typically be warranted.

\section{Upper Eyelid Elevation (MRD-I)}

On treatment days 1, 14, and 42, mean MRD-1 increased from baseline pre-instillation levels after oxymetazoline $0.1 \%$ instillation, with more modest increases after vehicle instillation (Figure 1). Notably, on all treatment days evaluated, mean MRD-1 increased over baseline at the earliest time point assessed ( 5 minutes post-instillation, assessed in Study RVL-1201-202) and tended to peak, among the time points evaluated, 2-6 hours post-instillation. On treatment days 1,14 , and 42 , mean MRD-1 at 5 minutes postinstillation was $1.64 \pm 0.88 \mathrm{~mm}, 1.80 \pm 1.03 \mathrm{~mm}$, and $1.88 \mathrm{~mm} \pm 0.95 \mathrm{~mm}$, respectively, among subjects receiving oxymetazoline $0.1 \%$. Mean MRD- 1 at the corresponding time points in the vehicle group were $1.30 \pm 0.80 \mathrm{~mm}$, $1.49 \pm 0.85 \mathrm{~mm}$, and $1.49 \pm 0.82 \mathrm{~mm}$, respectively.

Consistent with the observed change in mean MRD-1, once-daily administration of oxymetazoline $0.1 \%$ resulted in a significantly greater change from baseline than vehicle. As previously reported, both studies met their predefined secondary efficacy endpoint, with oxymetazoline $0.1 \%$ treatment resulting in a statistically significant increase in MRD-1 at 6 hours post-instillation on treatment day 1 and 2 hours post-instillation on treatment day 14 (both $\mathrm{p}<0.0001$ ). ${ }^{24}$ Examination of additional time point data here revealed a rapid and significant onset of action following oxymetazoline $0.1 \%$ administration. Oxymetazoline $0.1 \%$ significantly improved MRD-1 vs vehicle 5 minutes post-instillation on treatment days 1 , 14 , and 42 (all $\mathrm{p}<0.05$ ) (Figure 2). The mean change from baseline 5 minutes post-oxymetazoline $0.1 \%$ instillation (measured in Study RVL-1201-202) was $0.59 \pm 0.72 \mathrm{~mm}$

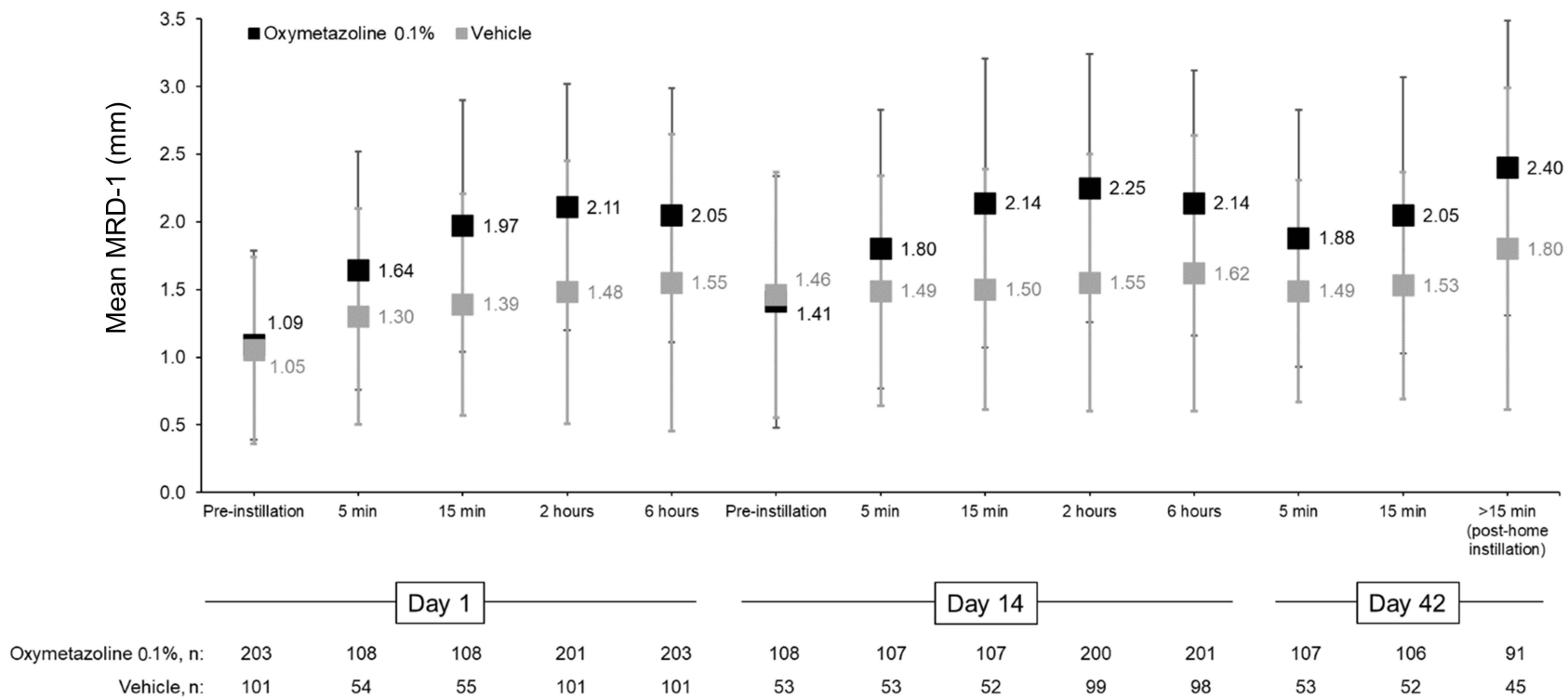

Figure I Mean (SD) marginal reflex distance I (MRD-I) at baseline and following once-daily administration (one drop per eye) of oxymetazoline $0.1 \%$ or vehicle, in patients with acquired ptosis. Five (5)- and 15-minute post-instillation data were collected in one study (RVL-I20I-202); >15-minute data on day 42 were collected in the other study (RVL-I20I-20I), in which the exact post-instillation timing of the day 42 evaluation was not pre-defined (subjects instilled their assigned treatment at home prior to going to the study site for their final study visit). $n=$ number of subjects evaluated (study eye only). 


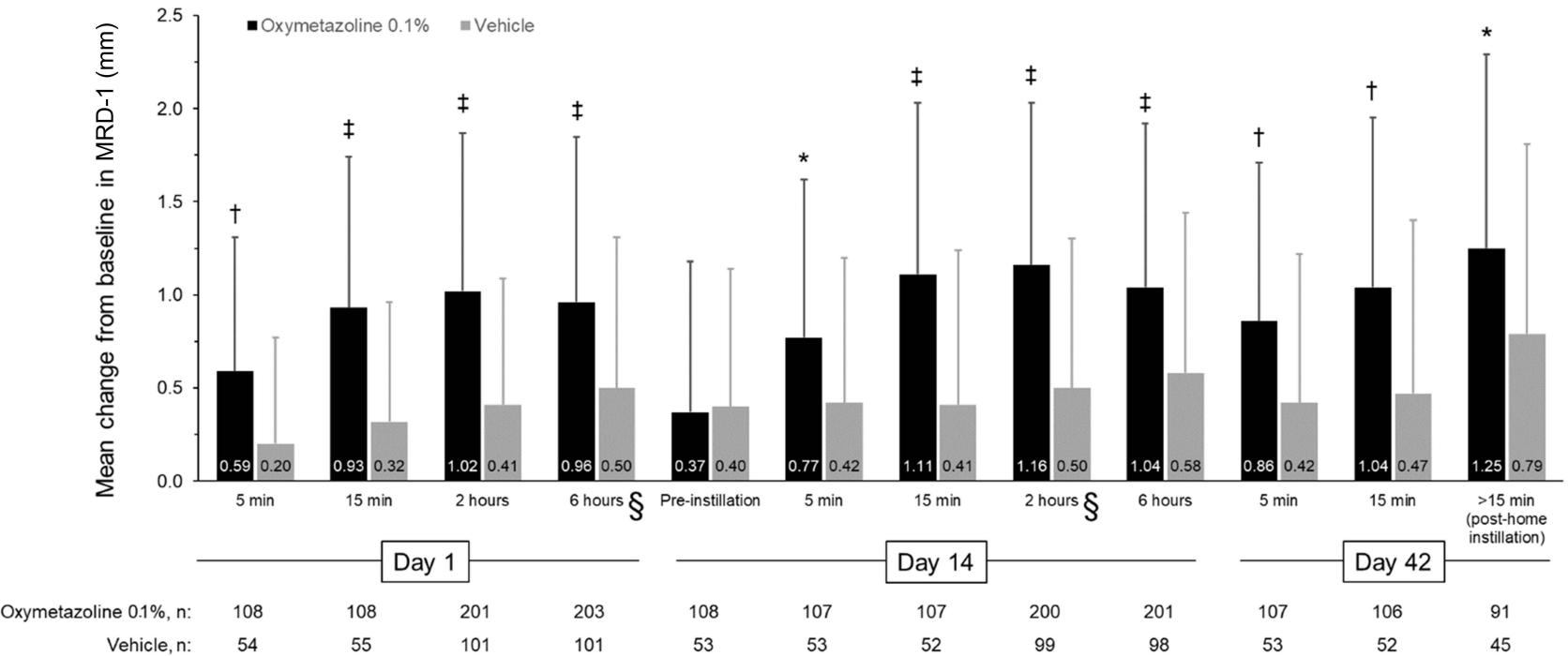

Figure 2 Mean (SD) change from baseline in marginal reflex distance I (MRD-I) following once-daily administration (one drop per eye) of oxymetazoline $0.1 \%$ or vehicle, in patients with acquired ptosis. ${ }^{*} p<0.05$ vs vehicle; ${ }^{\dagger} p<0.005$ vs vehicle; ${ }^{\ddagger} p<0.000$ I vs vehicle. ${ }^{\S}$ Data point adapted from Slonim et al. ${ }^{24}$ As previously reported, change from baseline in the pooled study population was significantly greater among subjects receiving oxymetazoline $0.1 \%$ than those receiving vehicle at the day I (6 hours) time point $(0.90 \pm 0.89 \mathrm{~mm}$ vs $0.50 \pm 0.8 \mathrm{Imm} ; \mathrm{p}<0.000 \mathrm{I})$ and the day I4 (2 hours) time point (I.16 $\pm 0.87 \mathrm{~mm}$ vs $0.50 \pm 0.80 \mathrm{~mm} ; \mathrm{p}<0.000 \mathrm{I}){ }^{24}$ Five $(5)$ - and I5-minute post-instillation data were collected in one study (RVL-I 20I-202); > I5-minute data on day 42 were collected in the other study (RVL-I20I-20I), in which the exact post-instillation timing of the day 42 evaluation was not pre-defined (subjects instilled their assigned treatment at home prior to going to the study site for their final study visit). $n=$ number of subjects evaluated (study eye only).

(vs $0.20 \pm 0.57 \mathrm{~mm}$ with vehicle, $\mathrm{p}=0.0007$ ) on treatment day $1,0.77 \pm 0.85 \mathrm{~mm}$ (vs $0.42 \pm 0.78 \mathrm{~mm}$ with vehicle; $\mathrm{p}=0.015)$ on treatment day 14 , and $0.86 \pm$ $0.85 \mathrm{~mm}$ (vs $0.42 \pm 0.80 \mathrm{~mm}$ with vehicle; $\mathrm{p}=0.0020$ ) on treatment day 42 . These statistically significant improvements with oxymetazoline $0.1 \%$ vs vehicle were sustained at 15 minutes, 2 hours, and 6 hours post-instillation on treatment days 1 and 14, as well as all time points assessed on day 42 in both studies (all $\mathrm{p}<0.05$ ) (Figure 2).

\section{Responder Analysis}

The proportion of patients showing improvement in MRD$1(>0 \%, \geq 50 \%$, or $\geq 90 \%$ improvement) was consistently greater in the oxymetazoline $0.1 \%$ group compared with vehicle throughout the study period (Figure 3), beginning after the first dose on treatment day 1. Five minutes following instillation on treatment day 1 (assessed in Study RVL-1201-202 only), $61.1 \%$ of subjects who received oxymetazoline $0.1 \%$ showed $>0 \%$ improvement (any measurable response), 50.9\% showed $\geq 50 \%$ improvement, and $38.0 \%$ showed $\geq 90 \%$ improvement in MRD- 1 . In comparison, the respective values for subjects in the vehicle group at this time point were $38.9 \%, 29.6 \%$, and $22.2 \%$. Response rates five minutes after oxymetazoline $0.1 \%$ instillation were similar on treatment days 14 and 42 (time points also assessed in Study RVL-1201-202 only).
The proportions of subjects showing a $>0 \%, \geq 50 \%$, and $\geq 90 \%$ improvement in MRD-1 five minutes postoxymetazoline $0.1 \%$ instillation on treatment day 14 were $68.2 \%, 57.9 \%$, and $41.1 \%$, respectively (vs $50.9 \%$ $43.4 \%$, and $35.8 \%$ with vehicle). Five minutes postinstillation on treatment day $42,72.0 \%$ of subjects receiving oxymetazoline $0.1 \%$ showed $>0 \%$ improvement, while $64.5 \%$ showed $\geq 50 \%$ improvement, and $43.0 \%$ showed $\geq 90 \%$ improvement in MRD-1. The respective values for vehicle at this time point were $54.7 \%, 39.6 \%$, and $30.2 \%$.

Pooled study results obtained 2 and 6 hours after treatment administration revealed similar results. On treatment days 1 and $14,>80 \%$ of subjects had a positive $(>0 \%$ increase in MRD-1) response 2 hours post-oxymetazoline $0.1 \%$ administration (vs 47.5 and $53.5 \%$ on treatment days 1 and 14, respectively, in the vehicle group). Response rates were comparable at 6 hours post-instillation on treatment days 1 and 14 , demonstrating that $>75 \%$ of subjects had a positive ( $>0 \%$ increase in MRD- 1 ) response in eyelid elevation 6 hours after administering oxymetazoline $0.1 \%$ (vs 52.5 and $55.1 \%$ on treatment days 1 and 14 , respectively, in the vehicle group).

Pooled day 42 data (comprising 5-minute postinstillation data from subjects in Study RVL-1201-202 and data from subjects in Study RVL-1201-201) showed that among subjects in the oxymetazoline $0.1 \%$ group, 


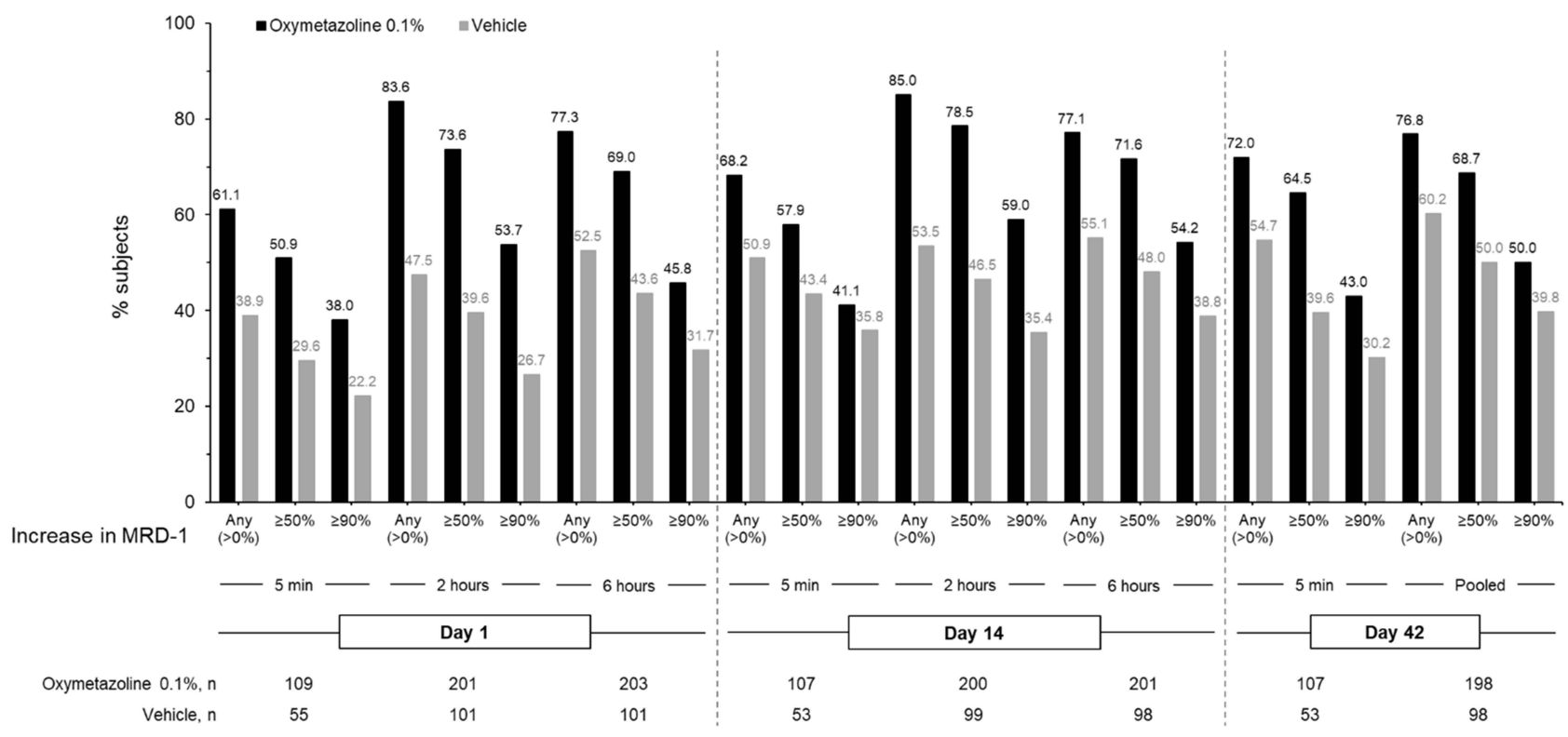

Figure 3 Proportion of patients with improvement in marginal reflex distance I (MRD-I) following once-daily administration (one drop per eye) of oxymetazoline $0.1 \%$ or vehicle, treatment day I (5 min, 2 hours, 6 hours), day 14 (5 min, 2 hours, 6 hours), day 42 (5 min, pooled). Five (5)- and I5-minute post-instillation data were collected in one study (RVL-I20I-202); > 15-minute data on day 42 were collected in the other study (RVL-I20I-20I), in which the exact post-instillation timing of the day 42 evaluation was not pre-defined (subjects instilled their assigned treatment at home prior to going to the study site for their final study visit). Day 42 pooled data comprise day 42 data from Study RVL-I20I-20I and 5-minute post-instillation data on day 42 from Study RVL-120I-202. n=number of subjects evaluated (study eye only).

$76.8 \%$ had $>0 \%$ improvement, $68.7 \%$ had a $\geq 50 \%$ improvement, and $50.0 \%$ had a $\geq 90 \%$ improvement in MRD-1. Corresponding numbers among subjects receiving vehicle were $60.2 \%, 50.0 \%$, and $39.8 \%$.

Overall, the proportion of subjects showing a positive response ( $>0 \%$ increase in MRD-1) to oxymetazoline $0.1 \%$ at any time point on treatment days 1,14 , or 42 was $>15 \%$ greater than the proportion of subjects showing a positive response after administering vehicle (range 16.6-36.1\% difference, with the largest differences typically seen 2 and 6 hours post-instillation).

\section{Discussion}

Improvement in MRD-1 with oxymetazoline $0.1 \%$ has been previously shown in parallel with significant improvement in superior visual field deficits. ${ }^{24}$ The indepth analysis of upper eyelid elevation presented here expands on these findings, demonstrating that once-daily administration of oxymetazoline $0.1 \%$ raised the upper eyelid in a rapid manner over at least 42 days of use. While change in MRD-1 was previously reported at the predefined secondary endpoints common to both studies, ${ }^{24}$ the present analysis of additional post-instillation data, including the 5- and 15-minute time points collected in Study RVL-1201-202, shows statistically significant improvement in MRD-1 as early as 5 minutes post- instillation on all three days examined (days 1, 14, and 42). Further, the observed magnitude of effect was consistent across time points, including when comparing 5and 15-minute time points to previously reported efficacy time points ( 6 hours post-instillation on day 1; 2 hours post-instillation on day 14). This significant effect of oxymetazoline $0.1 \%$ was observed in comparison to vehicle, which showed modest mean increases and lower overall response rates. The modest increases in MRD-1 in the group treated with vehicle may reflect some measure of normal variation occurring on the assessment days or a "placebo effect" in patients expecting upper eyelid elevation with treatment.

The observed response rates to oxymetazoline $0.1 \%$ are likewise encouraging. Though the degree of response for each subject is relative to that individual's baseline MRD1 , the high response rates indicate that the significant mean increases observed with oxymetazoline $0.1 \%$ treatment are not likely to be a product of large responses in a small number of individuals. The consistency of response rates across days and time points also indicates sustained responsiveness over the study period.

Ptosis must typically be relatively severe (eg, MRD-1 $\leq 2.0 \mathrm{~mm}$ ) to qualify for surgical correction for functional reasons. ${ }^{14}$ The subjects enrolled in the phase 3 oxymetazoline $0.1 \%$ studies represent a population for whom surgical 
correction might typically be considered. Based on the results from these studies, oxymetazoline $0.1 \%$ represents a potentially valuable non-invasive treatment option for individuals regardless of whether or not they might be candidates for surgery. This is important given that surgery can be associated with risks for bleeding, infection, mild keratitis, corneal abrasion, eyelid crease abnormalities, and distortion of the eyelid margin contour, ${ }^{6}$ and can require revision in the event of under- or over-correction. ${ }^{6,15}$ There can also be significant recovery time required subsequent to ptosis surgery, ${ }^{6}$ making it a less than optimal therapeutic option for some patients. Still, surgical approaches typically offer the greatest level of control over eyelid position, and when necessary, can reliably be used to provide increases in MRD-1 exceeding the mean increases reported here with oxymetazoline $0.1 \%$.

As previously reported for the pooled study cohort, oxymetazoline $0.1 \%$ has also been shown to have a favorable safety profile over 42 days of use, with adverse event (AE) rates comparable to those with vehicle (oxymetazoline $0.1 \%: 31.0 \%$ vs vehicle: $35.6 \%){ }^{24}$ The most common AEs (punctate keratitis [oxymetazoline 0.1\%: $5.4 \%$ vs vehicle: $3.0 \%$ ], blurred vision [oxymetazoline $0.1 \%: 3.4 \%$ vs vehicle: 0 ], conjunctival hyperemia [oxymetazoline $0.1 \%: 3.0 \%$ vs vehicle: $1.0 \%$ ], instillation site pain [oxymetazoline $0.1 \%: 3.0 \%$ vs vehicle: 0 ]) tended to occur early on in the course of treatment and were most often self-limited in nature. Neither treatment had any significant mean effects on vital signs (heart rate, blood pressure) or ophthalmic examination results, including intraocular pressure and pupil diameter, over 42 days of use. $^{24}$

MRD-1 represents, at least in part, the effect of ptosis that patients can observe. Reductions in MRD-1 are associated with impairment in the superior visual field ${ }^{9-12}$ and a visible effect on appearance that can result in psychological effects (ie, distress, anxiety, depression). ${ }^{13}$ Improvements in MRD-1, which reflect elevation of the upper eyelid, have the potential to alleviate these effects of ptosis. Further, measuring MRD-1 is an easily performed aspect of clinical examination/diagnosis, and serves as a quantitative measure that can be used to monitor progression or improvement of ptosis over time. ${ }^{25}$ MRD-1 measurement can therefore also allow for straightforward assessment of the effect of a rapidly acting, minimally invasive treatment, such as oxymetazoline $0.1 \%$.

The rapid effect of oxymetazoline $0.1 \%$ on upper eyelid elevation observed here is encouraging, though it should be noted that the early onset time points ( 5 and 15 minutes post-instillation) were only recorded in one of the two randomized trials analyzed here. From the perspective of the patient and practitioner, the observed rapid onset of oxymetazoline $0.1 \%$, while not permanent, may be desirable in comparison to the time required to prepare for, undergo, and recover from surgical intervention. Still, future studies elucidating the effect of a single dose of oxymetazoline $0.1 \%$ over a longer duration (for example, up to 12 or 24 hours) would further help inform clinical practice. Given the once-daily dosing regimen of oxymetazoline $0.1 \%$ and the fact that an individual will generally spend approximately 16 hours awake per day, data beyond 6 hours post-instillation will be informative. In addition, the present analysis was based on data from two 6-week studies, and the efficacy of treatment beyond the immediate post-instillation period on day 42 has not yet been evaluated. No instances of tachyphylaxis were observed in either study, ${ }^{24}$ and the significant improvement in upper eyelid elevation demonstrated on treatment day 42 , as well as consistent response rates across treatment days (Figures 2 and 3), supports the efficacy of oxymetazoline $0.1 \%$ over at least 6 weeks of use. Importantly, however, the efficacy of oxymetazoline $0.1 \%$ was not evaluated throughout day 42 (for example, at pre-defined 2- or 6-hour time points). Thus, the duration of effect of a single dose beyond 2 weeks of daily use remains to be fully understood. Evidence of tachyphylaxis with other nasal and ophthalmic $\alpha$-adrenergic agonists ${ }^{26-29}$ suggests that further study of the long-term efficacy, as well as safety, associated with chronic oxymetazoline $0.1 \%$ use is warranted. The results of a phase 3 randomized, doublemasked, placebo-controlled clinical safety study 12 weeks in duration (NCT03536949) will provide important insight into the safety of oxymetazoline $0.1 \%$, as well as patientreported outcomes, over a longer duration of use. In addition, while the improvements in MRD-1 from baseline observed here were significant, mean MRD-1 at any given post-instillation time point after oxymetazoline $0.1 \%$ use ranged from $1.64 \pm 0.88 \mathrm{~mm}$ to $2.40 \pm$ $1.09 \mathrm{~mm}$ (Figure 1), implying that a proportion of subjects still had ptosis in the moderate-to-severe range following treatment. Importantly, these mean values include subjects with no measurable response, which comprised $15.0 \%$ to $38.9 \%$ of subjects, depending on the time point (Figure 3 ). It will be important for future studies to evaluate the effect of oxymetazoline $0.1 \%$ in patients with less severe ptosis. Finally, as noted above, successful surgical intervention 
for ptosis has been shown to positively impact patient QoL. ${ }^{14,30}$ This study was not designed to assess QoL outcomes or to assess follow-up treatment (for example, the decision to pursue surgical correction); however, examination of potential QoL impacts associated with oxymetazoline $0.1 \%$ use would be of great interest, particularly given its minimally invasive nature.

\section{Conclusions}

In summary, this analysis of phase 3 data reveals that once-daily topical oxymetazoline $0.1 \%$ administration resulted in a rapid improvement in MRD-1 that was observed over 42 days. Combined with data supporting the ability of oxymetazoline $0.1 \%$ to improve visual function and its reported safety profile when used daily for 6 weeks, ${ }^{24}$ the present evidence suggests that this therapeutic approach may offer an effective, a non-surgical treatment option for patients with acquired blepharoptosis.

\section{Data Sharing Statement}

The authors do not intend to share individual deidentified study participant data.

\section{Acknowledgments}

Studies reported were funded by RVL Pharmaceuticals, Inc., an affiliate of Osmotica Pharmaceuticals plc (Bridgewater, NJ, USA). Editorial and administrative support was provided by BioScience Communications (New York, NY, USA) through funding provided by Osmotica Pharmaceuticals.

\section{Disclosure}

Jason Bacharach reports speaker fees from Aerie Pharmaceuticals, Alcon, Allergan, Bausch \& Lomb, Glaukos, New World Medical, and Sun Pharmaceutical Industries, Inc; consultant fees from Aerie Pharmaceuticals, Alcon, Allergan, Bausch \& Lomb, Injectsense, New World Medical, Optovue, and Osmotica Pharmaceuticals; personal fees from Sun Pharmaceutical Industries, Inc.; research support from Aerie Pharmaceuticals, Allergan, Novartis, Glaukos, Optovue, and Ocular Therapeutix. David L. Wirta reports research support from RVL Pharmaceuticals, Inc., and Osmotica Pharmaceuticals. Robert Smyth-Medina reports personal fees from Sun Pharmaceutical Industries, Inc.; research support from Aerie Pharmaceuticals, Alcon, Allergan, Aurinia, Auven, Bausch \& Lomb, Eleven, Encore, Evidera, Eyegate, Hi Tech Pharmacal, Inotek, Inspire,
Ista, Kala, Novartis, OmegaD, Ono, OTX, RVL Pharmaceuticals, Inc., Santen, SARcode, Shire, Silk, Senju, Valeant, and Xigen. Michael S. Korenfeld reports consultant fees from Osmotica Pharmaceuticals. Shane R. Kannarr reports personal fees from Allergan, Alcon, Bausch \& Lomb, Essilor, Johnson \& Johnson, Novartis, Oculos, Optovue, and Osmotica Pharmaceuticals. Shane Foster reports consultant and speaker fees from Osmotica Pharmaceuticals. Mark J Jaros reports consultant fees from Osmotica Pharmaceuticals. Charles B. Slonim reports research support and consultant fees from RVL Pharmaceuticals, Inc. and Osmotica Pharmaceuticals. The authors report no other conflicts of interest in this work.

\section{References}

1. Fausett BV, Nerad JA. Upper eyelid ptosis and retraction. In: Fay A, Dolman PJ, editors. Diseases and Disorders of the Orbit and Ocular Adnexa: Expert Consult. 1st ed. St Louis: Elsevier; 2016:525-539.

2. Forman WM, Leatherbarrow B, Sridharan GV, Tallis RC. A community survey of ptosis of the eyelid and pupil size of elderly people. Age Ageing. 1995;24:21-24. doi:10.1093/ageing/24.1.21

3. Hashemi H, Khabazkhoob M, Emamian MH, et al. The prevalence of ptosis in an Iranian adult population. $J$ Curr Ophthalmol. 2016;28:142-145. doi:10.1016/j.joco.2016.04.005

4. Kim MH, Cho J, Zhao D, et al. Prevalence and associated factors of blepharoptosis in Korean adult population: the Korea National Health and Nutrition Examination Survey. Eye. 2017;31:940-946. doi:10. 1038/eye.2017.43

5. Lim JM, Hou JH, Singa RM, Aakalu VK, Setabutr P. Relative incidence of blepharoptosis subtypes in an oculoplastics practice at a tertiary care center. Orbit. 2013;32:231-234. doi:10.3109/0167 6830.2013.788673

6. Finsterer J. Ptosis: causes, presentation, and management. Aesthetic Plast Surg. 2003;27:193-204. doi:10.1007/s00266-003-0127-5

7. Latting MW, Huggins AB, Marx DP, Giacometti JN. Clinical evaluation of blepharoptosis: distinguishing age-related ptosis from masquerade conditions. Semin Plast Surg. 2017;31:5-16. doi:10.1055/ s-0037-1598188

8. Sudhakar P, Vu Q, Kosoko-Lasaki O, Palmer M. Upper eyelid ptosis revisited. Am J Clin Med. 2009;6:5-14.

9. Alniemi ST, Pang NK, Woog JJ, Bradley EA. Comparison of automated and manual perimetry in patients with blepharoptosis. Ophthal Plast Reconstr Surg. 2013;29:361-363. doi:10.1097/IOP.0b013e3 1829a7288

10. Cahill KV, Burns JA, Weber PA. The effect of blepharoptosis on the field of vision. Ophthal Plast Reconstr Surg. 1987;3:121-125. doi:10.1097/00002341-198703030-00001

11. McKean-Cowdin R, Varma R, Wu J, Hays RD, Azen SP; Los Angeles Latino Eye Study Group. Severity of visual field loss and health-related quality of life. Am $J$ Ophthalmol. 2007;143: 1013-1023. doi:10.1016/j.ajo.2007.02.022

12. Meyer DR, Stern JH, Jarvis JM, Lininger LL. Evaluating the visual field effects of blepharoptosis using automated static perimetry. Ophthalmology. 1993;100:651-658. doi:10.1016/S0161-6420(93)31 593-9

13. Richards HS, Jenkinson E, Rumsey N, et al. The psychological well-being and appearance concerns of patients presenting with ptosis. Eye (Lond). 2014;28:296-302. doi:10.1038/eye.2013.264 
14. Cahill KV, Bradley EA, Meyer DR, et al. Functional indications for upper eyelid ptosis and blepharoplasty surgery: a report by the American Academy of Ophthalmology. Ophthalmology. 2011;118:2510-2517. doi:10.1016/j.ophtha.2011.09.029

15. Chou E, Liu J, Seaworth C, et al. Comparison of revision rates on anterior- and posterior-approach ptosis surgery: a retrospective review of 1519 cases. Ophthal Plast Reconstr Surg. 2018;34:246-253. doi:10.1097/IOP.0000000000000938

16. Haenisch B, Walstab J, Herberhold S, et al. Alpha-adrenoceptor agonistic activity of oxymetazoline and xylometazoline. Fundam Clin Pharmacol. 2010;24:729-739. doi:10.1111/j.1472-8206.2009.00805.x

17. Afrin ${ }^{\circledR}$ original oxymetazoline decongestant nasal spray [package insert]. Whippany, NJ: Bayer Healthcare LLC; 2017.

18. Druce HM, Ramsey DL, Karnati S, Carr AN. Topical nasal decongestant oxymetazoline $(0.05 \%)$ provides relief of nasal symptoms for 12 hours. Rhinology. 2018;56:343-350. doi:10.4193/Rhin17.150

19. Duzman E, Anderson J, Vita JB, Lue JC, Chen CC, Leopold IH. Topically applied oxymetazoline. Ocular vasoconstrictive activity, pharmacokinetics, and metabolism. Arch Ophthalmol. 1983;101:1122-1126. doi:10.1001/archopht.1983.01040020124022

20. Visine L.R. (oxymetazoline hydrochloride 0.025\%) [package insert]. New Brunswick, NJ: Johnson \& Johnson Healthcare Products, Inc; 2016.

21. Beard C. Müller's superior tarsal muscle: anatomy, physiology, and clinical significance. Ann Plast Surg. 1985;14:324-333. doi:10.1097/ 00000637-198504000-00005

22. Esmaeli-Gutstein B, Hewlett BR, Pashby RC, Oestreicher J, Harvey JT. Distribution of adrenergic receptor subtypes in the retractor muscles of the upper eyelid. Ophthal Plast Reconstr Surg. 1999;15:92-99. doi:10.1097/00002341-199903000-00005
23. Hauck MJ, Steele EA, Perry CB. Predictability of the phenylephrine test with regard to eyelid skin appearance in patients who undergo Muller muscle-conjunctival resection without blepharoplasty. Ophthal Plast Reconstr Surg. 2019;36:191-193. doi:10.1097/IOP. 0000000000001510

24. Slonim CB, Foster S, Jaros M, et al. Association of oxymetazoline hydrochloride, $0.1 \%$, solution administration with visual field in acquired ptosis: a pooled analysis of 2 randomized clinical trials. JAMA Ophthalmol. 2020;138:1168-1175. doi:10.1001/jamaophthalmol.2020. 3812

25. Putterman AM, Urist MJ. Müller muscle-conjunctiva resection. Technique for treatment of blepharoptosis. Arch Ophthalmol. 1975;93:619-623. doi:10.1001/archopht.1975.01010020595007

26. Ramey JT, Bailen E, Lockey RF. Rhinitis medicamentosa. J Investig Allergol Clin Immunol. 2006;16:148-155.

27. Vaidyanathan S, Williamson P, Clearie K, Khan F, Lipworth B. Fluticasone reverses oxymetazoline-induced tachyphylaxis of response and rebound congestion. Am J Respir Crit Care Med. 2010;182:19-24. doi:10.1164/rccm.200911-1701OC

28. Soparkar CN, Wilhelmus KR, Koch DD, Wallace GW, Jones DB. Acute and chronic conjunctivitis due to over-the-counter ophthalmic decongestants. Arch Ophthalmol. 1997;115:34-38. doi:10.1001/archopht. 1997.01100150036004

29. Spector SL, Ralzman MB. Conjunctivitis medicamentosa. J Allergy Clin Immunol. 1994;94:134-136. doi:10.1016/0091-6749(94)90081-7

30. Battu VK, Meyer DR, Wobig JL. Improvement in subjective visual function and quality of life outcome measures after blepharoptosis surgery. Am J Ophthalmol. 1996;121:677-686. doi:10.1016/S00029394(14)70634-8
Clinical Ophthalmology

\section{Publish your work in this journal}

Clinical Ophthalmology is an international, peer-reviewed journal covering all subspecialties within ophthalmology. Key topics include: Optometry; Visual science; Pharmacology and drug therapy in eye diseases; Basic Sciences; Primary and Secondary eye care; Patient Safety and Quality of Care Improvements. This journal is indexed on PubMed

Submit your manuscript here: https://www.dovepress.com/clinical-ophthalmology-journal

\section{Dovepress}

Central and CAS, and is the official journal of The Society of Clinical Ophthalmology (SCO). The manuscript management system is completely online and includes a very quick and fair peer-review system, which is all easy to use. Visit http://www.dovepress.com/ testimonials.php to read real quotes from published authors. 